\title{
Emergence of the Second Law out of Reversible Dynamics
}

\author{
L.G. Van Willigenburg • W.L. De Koning
}

Received: 25 September 2008 / Accepted: 18 August 2009 / Published online: 3 September 2009

(C) The Author(s) 2009. This article is published with open access at Springerlink.com

\begin{abstract}
If one demystifies entropy the second law of thermodynamics comes out as an emergent property entirely based on the simple dynamic mechanical laws that govern the motion and energies of system parts on a micro-scale. The emergence of the second law is illustrated in this paper through the development of a new, very simple and highly efficient technique to compare time-averaged energies in isolated conservative linear large scale dynamical systems. Entropy is replaced by a notion that is much more transparent and more or less dual called ectropy. Ectropy has been introduced before but we further modify the notion of ectropy such that the unit in which it is expressed becomes the unit of energy. The second law of thermodynamics in terms of ectropy states that ectropy decreases with time on a large enough timescale and has an absolute minimum equal to zero. Zero ectropy corresponds to energy equipartition. Basically we show that by enlarging the dimension of an isolated conservative linear dynamical system and the dimension of the system parts over which we consider time-averaged energy partition, the tendency towards equipartition increases while equipartition is achieved in the limit. This illustrates that the second law is an emergent property of these systems. Finally from our large scale linear dynamic model we clarify Loschmidt's paradox concerning the irreversible behavior of ectropy obtained from the reversible dynamic laws that govern motion and energy at the micro-scale.
\end{abstract}

W.L. De Koning retired from the Department of Mathematics of Delft University of Technology.

L.G. Van Willigenburg $(\bowtie)$

Systems \& Control Group, Wageningen University, P.O. Box 17, 6700AA Wageningen,

The Netherlands

e-mail: gerard.vanwilligenburg@wur.nl

W.L. De Koning

Kroeskarper 6, Leiden, The Netherlands

e-mail: wilros@planet.nl 
Keywords Ectropy $\cdot$ Entropy fluctuations $\cdot$ Hamiltonian systems $\cdot$ Reversible dynamics $\cdot$ Emergent properties $\cdot$ Second law of thermodynamics

\section{Introduction}

The second law of thermodynamics has puzzled and is puzzling scientists for more than a century. Maxwell's Demon is probably the best advocate of the puzzle. Thermodynamics itself also suffers from controversies. Quoting the leading system scientist Jan Willems from a book review on thermodynamics [1]: "From a pedagogical point of view, thermodynamics is a disaster." He goes on to mention the main stumbling blocks as being the often inconsistent use of mathematics to describe thermodynamics, entropy being an unmeasurable quantity that somehow has to be deduced from the system laws, the use of statistical arguments at random moments in thermodynamic reasoning and the supposed metaphysical nature of the second law. Fortunately Jan Willems concluded that the book under review by Haddad et al. [2] did not suffer from all these stumbling blocks. The book actually takes a dynamical systems approach to thermodynamics. It is free of any statistical arguments and introduces ectropy as an alternative much more transparent quantity to entropy. Interestingly, but also somewhat disappointingly, the second law of thermodynamics is introduced as an axiom.

Our research into thermodynamics and the second law was initiated by an unexpected request to teach a small course on thermodynamics. Being system scientists ourselves the book review of Jan Willems and the book itself by Haddad et al. inspired our recent research reported in this paper that also takes a dynamical systems approach. The main inspiration however was our hypothesis that the second law of thermodynamics should have a "Darwinian explanation" i.e. it should require no more than the simple dynamic laws governing the system parts and their interaction at the micro-scale.

Although our research is restricted to linear large scale dynamical systems we believe it clarifies the puzzles surrounding the second law of thermodynamics. Our approach differs from most of the analysis and work performed by physicists in that no statistics are used to describe the system. Everything just follows from the simple, deterministic, reversible, dynamic laws governing the motion and energy of all system parts at the micro-scale. In this respect our work is similar to that of Bernstein and Bhat [3], Bhat and Bernstein [4] and Rapisarda and Willems [5]. What distinguishes our work from theirs is (1) the use of a new, highly efficient computational scheme to compare time-averaged energies and (2) the linking of growing system dimensions to the emergence of the second law of thermodynamics. To investigate the emergence, systems with identical parts on the micro-scale will be considered. In that case equipartition of temperature equals equipartition of energy over macroscopic system parts that are (approximately) identical. The extension to equipartition of temperature is made by considering unequal macroscopic system parts and their thermal capacities.

Statistical mechanics is generally invoked to try to describe approximately the macroscopic behavior of large sets of microscopic particles. Statistical mechanics 
uses statistics and probability measures. Both of these do not appear anywhere in our system description and computations. We only argue from the outcome of our computations that the probability for certain things to happen is either very high or very low. Also to show that several results are independent of initial conditions we will perform our computations with different initial conditions having certain properties. Apart from these properties the initial conditions are selected randomly. In justifying statistical mechanics the ergodic hypothesis is believed to play a central role [6]. Roughly speaking it assumes that time-averages and ensemble averages of dynamical systems become equal as time tends to infinity. Although we will compute timeaverages of dynamical systems these computations do not require this hypothesis to be satisfied. Also equilibrium conditions are generally required to justify statistical mechanics [7]. They are also not needed to obtain our results.

To arrive at our results the use of a specific type of system description turns out very convenient. Section 2 describes how general mass-spring systems are represented in this format. Next in Sect. 3 ectropy and energy equipartition are introduced. The systems considered in this paper will all be of the mass-spring type. Moreover they will all be diagonalizable i.e. all eigenvalues are assumed to be distinct (they are all imaginary due to the conservative nature of the system). As described in Sect. 4 such systems have normal mode representations that are the key to the new, very efficient computation and comparison of time-averaged energies presented in Sects. 5 and 6. These computations are finally exploited in Sect. 7 of this paper to illustrate the emergence of the second law. The masses and springs represent the microscopic level of the system. As their number increases a macroscopic level emerges to which the second law applies.

Normal modes of linear systems are decoupled and do not exchange energy. Therefore the linear mass-spring systems considered in this paper might be considered a worst case for the second law of thermodynamics to hold. In Sect. 8 we clarify Loschmidt's paradox concerning the irreversible behavior of ectropy obtained from the reversible dynamic laws that govern motion and energy at the micro-scale. Interpretations, conclusions and speculations concerning the results put forward in this paper are finally presented in Sect. 9.

\section{Hamiltonian Description of Mass-Spring Systems}

The second law applies to systems that are isolated (autonomous) and conserve energy (are conservative). Mass-spring systems are of this type. The analysis in this paper is restricted to this type of system because: (1) mass-spring systems are simple, well understood and therefore easy to interpret; (2) mass-spring systems are linear and admit a normal mode representation that enables a very efficient computation of time-averaged energy partition that circumvents the need for numerical integration; (3) due to their decoupled normal modes mass-spring systems may be considered a worst case for the second law of thermodynamics to hold.

Consider a one-dimensional mass-spring system in which all masses and springs have one degree of freedom, say moving from left to right and vice-versa. To further describe the system consider it to be in equilibrium. The system consists of $n$ masses $m_{i}, i=1,2, \ldots, n$, numbered from left to right, and $s$ linear springs with spring 


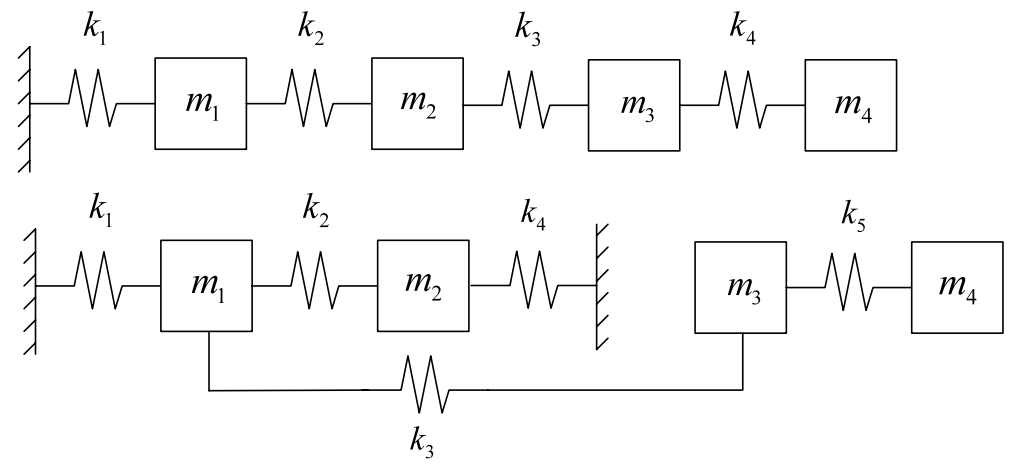

Fig. 1 Two examples of 1-dimensional mass-spring systems

constants $k_{i}, i=1,2, \ldots, s$, that interconnect these masses in some manner. Figure 1 represents two examples of such a system.

To describe the equations of motion of such a system let $p_{i}(t), i=1,2, \ldots, n$, denote the shifts of the masses from their equilibrium positions at any time $t$. It will be convenient to use a Hamiltonian state-space representation of the system. This representation consists of a series of first-order differential equations represented using matrices and vectors. To obtain this representation introduce the column vector

$$
x(t)=\left[\begin{array}{llllll}
p_{1}(t) & \ldots & p_{n}(t) & \dot{p}_{1}(t) & \ldots & \dot{p}_{n}(t)
\end{array}\right]^{\mathrm{T}} \in R^{2 n}
$$

where the superscript $T$ denotes transpose. The column vector $x$ in (2.1) is called the state vector of the system. It contains the state-variables of the system being the shifts of the masses from their equilibrium positions as well as their velocities. The equations of motion of the system can now be represented in the following form:

$$
\dot{x}(t)=A x(t), \quad A=\left[\begin{array}{cc}
0_{n} & I_{n} \\
-\operatorname{diag}\left(\frac{1}{m_{1}}, \frac{1}{m_{2}}, \ldots, \frac{1}{m_{n}}\right) K_{s}^{\mathrm{T}} K_{s} & 0_{n}
\end{array}\right] \in R^{2 n \times 2 n}
$$

In (2.2) $I_{n}$ denotes the identity matrix of dimension $n, 0_{n}$ a square zero matrix of dimension $n, \operatorname{diag}\left(\frac{1}{m_{1}}, \frac{1}{m_{2}}, \ldots, \frac{1}{m_{n}}\right)$ a diagonal square matrix of dimension $n$ with diagonal elements $\frac{1}{m_{1}}, \frac{1}{m_{2}}, \ldots, \frac{1}{m_{n}}$ respectively. The matrix $K_{s} \in R^{s \times n}$ represents the influence of the springs. If spring $i=1,2, \ldots, s$ connects the masses $m_{j(i)}, m_{k(i)}$, $1 \leq j(i)<k(i) \leq n$, then row $i$ of the matrix $K_{s}$ is identically zero except for element $j(i)$ that equals $\sqrt{k_{i}}$ and element $k(i)$ that equals $-\sqrt{k_{i}}$. If a spring on one side connects to a reference wall then this wall must be treated as an infinite mass. Therefore we may set to zero the corresponding element in $K_{s}$. As a result the reference walls may be left out of the description and the sign of the single non-zero element that is left over in row $i$ is positive if the wall is to the right and negative if the wall is to the left of the other mass to which the spring connects.

The total energy in the system, denoted by $H$, which is also called the Hamiltonian of the system, equals the sum of all potential and kinetic energies,

$$
H=\frac{1}{2} \sum_{i=1}^{n} m_{i} \dot{p}_{i}^{2}+\frac{1}{2} \sum_{i=1}^{s} k_{i} l_{i}^{2}
$$


In (2.3) $l_{i}=p_{j(i)}-p_{k(i)}, i=1,2, \ldots, s$, represent the stretches of the springs. They can have either sign and are zero in equilibrium. An alternative compact representation of (2.3) is

$$
H=\frac{1}{2} x^{\mathrm{T}} Q x, \quad Q=\left[\begin{array}{cc}
K_{s}^{\mathrm{T}} K_{s} & 0 \\
0 & \operatorname{diag}\left(m_{1}, m_{2}, \ldots, m_{n}\right)
\end{array}\right] \in R^{2 n \times 2 n}
$$

\section{Ectropy, Energy Equipartition and the Second Law}

Ectropy is introduced in this section as an alternative more transparent quantity to entropy. Ectropy is a measure of how much energies associated to system parts differ. If all the energies are equal this is called energy equipartition. In that case the ectropy measure equals its absolute minimum of zero. To investigate the second law starting from the dynamics at the micro-scale, instead of energies associated to system parts we will consider time-averaged energies in the limit as time goes to infinity. This averages out the very fast fluctuations of energy at the micro-scale and enables an analysis on a macroscopic time-scale. This type of analysis is required since the second law of thermodynamics applies only to large enough (macroscopic) time-scales and large enough (macroscopic) parts of a system.

The definition of ectropy requires a separation of the system into parts that together make up the whole system. To investigate the second law of thermodynamics these parts are macroscopic physical parts of the system that are spatially connected. According to the second law of thermodynamics the temperature differences between such parts tend to zero in the long run. If the macroscopic system parts are identical temperature differences may be replaced by energy differences. Ectropy measures the difference of energies captured by system parts.

In this paper we consider mass-spring systems. To investigate the second law, initially identical system parts will be considered. These parts contain an (almost) equal number of masses and springs that are connected to each other. Presume the mass-spring system (2.2) is separated in this manner into $N$ parts. Let,

$$
E(t)=\left[\begin{array}{llll}
E_{1}(t) & E_{2}(t) & \ldots & E_{N}(t)
\end{array}\right]^{\mathrm{T}}
$$

denote the associated energy vector where $E_{k}(t)$ represents the sum of all kinetic and potential energies contained by system part $k=1,2, \ldots, N$. Since the energy in the system is conserved,

$$
\sum_{k=1}^{N} E_{k}(t)=H
$$

The momentary energy partitioning $E(t)$ in (3.1) is an equipartition if,

$$
\forall k, j \in\{1,2, \ldots, N\}: \quad E_{k}(t)=E_{j}(t)
$$

The ectropy $U(t)$ associated to the momentary energy partitioning $E(t)$ in (3.1) is

$$
U(t)=\sum_{k=1}^{N}\left|E_{k}(t)-\bar{E}\right|, \quad \bar{E}=\frac{H}{N}
$$


Energy equipartition does not refer to momentary but to time-averaged energy partitionings.

Let

$$
\bar{E}_{k}=\lim _{T \rightarrow \infty} \frac{1}{T} \int_{0}^{T} E_{k}(t) d t, \quad k=1,2, \ldots, N
$$

denote the time-averaged values of $E_{k}(t), k=1,2, \ldots, N$. Energy equipartition occurs if,

$$
\forall x(0), \forall k, j \in\{1,2, \ldots, N\}: \quad \bar{E}_{k}=\bar{E}_{j}
$$

The next ectropy measure $U$ is similar to (3.4) but refers to time-averaged energies,

$$
U=\sum_{k=1}^{N}\left|\bar{E}_{k}-\bar{E}\right|, \quad \bar{E}=\frac{H}{N}
$$

In this paper we consider isolated conservative linear systems with identical parts on a micro-scale. For such systems the second law of thermodynamics states that the ectropy $U(t)$ associated to identical large enough (macroscopic) system parts $k=1,2, \ldots, N$ always decreases on a large enough time-scale (macroscopic timescale) and becomes zero in the limit as time tends to infinity.

In this paper we will present an efficient, accurate way to check whether ectropy decreases over arbitrary time intervals. But our focus will be on whether ectropy reaches the zero limit regardless of the initial conditions. From (3.2), (3.6) observe that ectropy as defined by (3.7) is a nonnegative measure of how far a system is removed from energy equipartition. Zero ectropy corresponds to energy equipartition. Also the unit of ectropy in both (3.4) and (3.7) equals the unit of energy.

\section{Normal Mode Representation and Energy Partition}

The normal mode representation of the linear system (2.2) enables a comparison of time-averaged energies such as those in (3.1) without the need to perform numerical integration. Numerical integration is expensive computationally and becomes highly inaccurate for systems with largely different time-scales such as the large scale massspring systems considered in this paper.

To arrive at the normal mode representation consider the eigenvalue decomposition of the square matrix $A$ in (2.2) represented by

$$
A V=V D, \quad V=\left[v_{1}, v_{2}, \ldots, v_{2 n}\right], D=\operatorname{diag}\left(\lambda_{1}, \lambda_{2}, \ldots, \lambda_{2 n}\right)
$$

In (4.1) $v_{i} \in C^{n}, i=1,2, \ldots, 2 n$ are the eigenvectors and $\lambda_{i} \in C^{1}, i=1,2, \ldots, 2 n$ the eigenvalues of $A$. Because the system preserves energy (is conservative) $v_{i}=$ $\bar{v}_{i+1}, i=1,3, \ldots, 2 n-1$, and $\lambda_{i}=\bar{\lambda}_{i+1}, i=1,3, \ldots, 2 n-1$, where the overbar denotes complex conjugate. Now build the following matrix:

$$
V^{\prime}=\left[v_{1}^{\prime}, v_{2}^{\prime}, \ldots, v_{2 n}^{\prime}\right] \in R^{2 n \times 2 n}, \quad v_{j}^{\prime} \in R^{2 n}, j=1,2, \ldots, 2 n
$$


with

$$
v_{j}^{\prime}=\operatorname{real}\left(v_{j}\right), \quad j=\operatorname{odd}, \quad v_{j}^{\prime}=\operatorname{imag}\left(v_{j}\right), \quad j=\text { even }
$$

Then if $A$ is diagonalizable and conservative (i.e. with only distinct pairs of complex conjugate imaginary eigenvalues and pairs of complex conjugate eigenvectors),

$$
A^{\prime}=V^{\prime-1} A V^{\prime} \in R^{2 n \times 2 n},
$$

exists and is an anti-symmetric block-diagonal matrix,

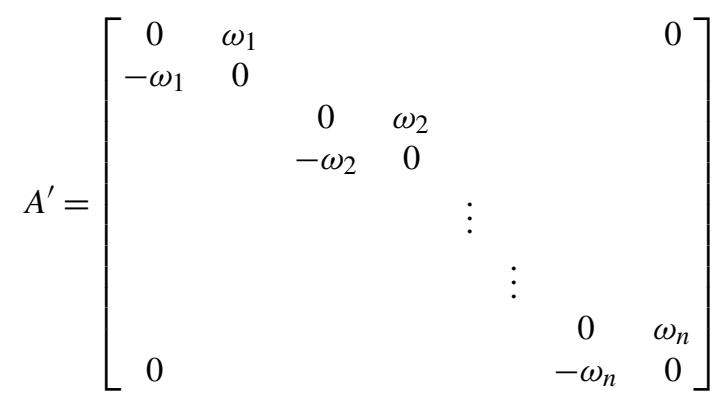

The transformation (4.4) is associated to a change of basis,

$$
V^{\prime} x^{\prime}=x
$$

of the system (2.2). The columns of $V^{\prime}$ represent the new basis vectors and $x^{\prime}$ is the state representation in this new state basis. The linear system,

$$
\dot{x}^{\prime}=A^{\prime} x^{\prime}, \quad x^{\prime} \in R^{2 n}
$$

obtained after this change of basis describes the behavior of the so called normal modes. Each normal mode $i=1,2, \ldots, n$, is described by the two consecutive states $x_{j}^{\prime}, x_{j+1}^{\prime}, j=2(i-1)+1$ of the state vector $x^{\prime}$. According to (4.5) they satisfy

$$
\left[\begin{array}{c}
\dot{x}_{j}^{\prime} \\
\dot{x}_{j+1}^{\prime}
\end{array}\right]=\left[\begin{array}{cc}
0 & \omega_{i} \\
-\omega_{i} & 0
\end{array}\right]\left[\begin{array}{c}
x_{j}^{\prime} \\
x_{j+1}^{\prime}
\end{array}\right]
$$

So the normal modes do not interact and therefore they do not exchange energy. In the new state basis the total energy $\frac{1}{2} x^{\mathrm{T}}(t) Q x(t)$ is represented by

$$
\frac{1}{2} x^{\prime \mathrm{T}}(t) Q^{\prime} x^{\prime}(t), \quad Q^{\prime}=V^{\prime \mathrm{T}} Q V^{\prime}
$$

where $Q^{\prime}$ is diagonal. Therefore the constant energy $E_{i}^{m}, i=1,2, \ldots, n$, associated to each normal mode is represented by

$$
\begin{aligned}
E_{i}^{m} & =\left[\begin{array}{ll}
x_{j}^{\prime}(t) & x_{j+1}^{\prime}(t)
\end{array}\right]\left[\begin{array}{cc}
Q_{j, j}^{\prime} & 0 \\
0 & Q_{j+1, j+1}^{\prime}
\end{array}\right]\left[\begin{array}{c}
x_{j}^{\prime}(t) \\
x_{j+1}^{\prime}(t)
\end{array}\right] \\
j & =2(i-1)+1, i=1,2, \ldots, n
\end{aligned}
$$


Each pair of columns $j, j+1, j=2(i-1)+1, i=1,2, \ldots, n$, of the transformation matrix $V^{\prime}$ in (4.4) has the following properties. Either (1) the last $n$ components of column $j$ are zero and the first $n$ components of column $j+1$ or (2) the first $n$ components of column $j$ are zero and the last $n$ components of column $j+1$. As a result in case (1) $x_{j}^{\prime}$ is a linear combination of $p_{i}, i=1,2, \ldots, n$, and $x_{j+1}^{\prime}$ a linear combination of $\dot{p}_{i}, i=1,2, \ldots, n$. Now all potential energy in the system depends solemnly on $p_{i}, i=1,2, \ldots, n$, and all kinetic energy depends solemnly on $\dot{p}_{i}, i=1,2, \ldots, n$. Then from (4.10),

$E_{i}^{m p}(t)=Q_{j, j}^{\prime} x_{j}^{\prime 2}(t), \quad E_{i}^{m k}(t)=Q_{j+1, j+1}^{\prime} x_{j+1}^{\prime 2}(t), \quad E_{i}^{m}=E_{i}^{m p}(t)+E_{i}^{m k}(t)$

where $E_{i}^{m p}(t), E_{i}^{m k}(t)$ denote respectively the potential and kinetic energy associated to normal mode $i=1,2, \ldots, n$. In case (2) $E_{i}^{m p}(t), E_{i}^{m k}(t)$ must be interchanged.

Equation (4.8) represents a harmonic oscillator and has the following solution:

$$
x_{j}^{\prime}(t)=A \sin \left(\omega_{i} t+\varphi\right), \quad x_{j+1}^{\prime}(t)=A \sin \left(\omega_{i} t+\varphi+\pi / 2\right), \quad t \geq 0
$$

where $A, \varphi$ depend on the initial state $\left[x_{j}^{\prime}(0) x_{j+1}^{\prime}(0)\right]^{\mathrm{T}}$ as follows:

$$
A=\sqrt{x_{j}^{\prime 2}(0)+x_{j+1}^{\prime 2}(0)}, \quad \varphi=\operatorname{atan} 2\left(x_{j}^{\prime}(0), x_{j+1}^{\prime}(0)\right)
$$

Due to (4.12), (4.13) the states of the normal mode representation (4.7), (4.5) have the following properties that play a central role in establishing energy equipartition,

$$
\begin{aligned}
& \frac{1}{2 \pi \omega_{i}} \int_{0}^{2 \pi / \omega_{i}} x_{j}^{\prime 2}(t) d t=\frac{1}{2 \pi \omega_{i}} \int_{0}^{2 \pi / \omega_{i}} x_{j+1}^{\prime 2}(t) d t=\frac{1}{2} A^{2} \\
&=\frac{1}{2}\left(x_{j}^{\prime 2}(0)+x_{j+1}^{\prime 2}(0)\right), \quad j=1,3, \ldots, 2 n-1 \\
& \frac{1}{2 \pi \omega_{i}} \int_{0}^{2 \pi / \omega_{i}} x_{j}^{\prime}(t) x_{j+1}^{\prime}(t) d t=0, \quad j=1,3, \ldots, 2 n-1
\end{aligned}
$$

Since all the normal mode frequencies are distinct i.e.,

$$
\forall i \neq k: \quad \omega_{i} \neq \omega_{k}, i, k \in\{1,2, \ldots, n\}
$$

we have

$$
\lim _{T \rightarrow \infty} \frac{1}{T} \int_{0}^{T} x_{j}^{\prime}(t) x_{k}^{\prime}(t) d t=0, \quad j \neq k, j, k \in\{1,2, \ldots, 2 n\}
$$

The normal modes do not interact and do not exchange energy. For a mass-spring system with $s=n=3$ Fig. 2 illustrates how every initial condition partitions its associated energy over the 3 normal modes. This is indicated as energy partitioning 1. By manipulating the initial state $x(0)$, the initial conditions $x_{j}^{\prime}(0), j=1,2, \ldots, 2 n$ of the normal modes may be manipulated arbitrarily. If the initial conditions of a normal mode are taken to be zero this mode is inactive because their states remain zero. Consider the 3 mass-spring pairs $m_{i}, k_{i}, i=1,2,3$, as represented on the right in Fig. 2. 


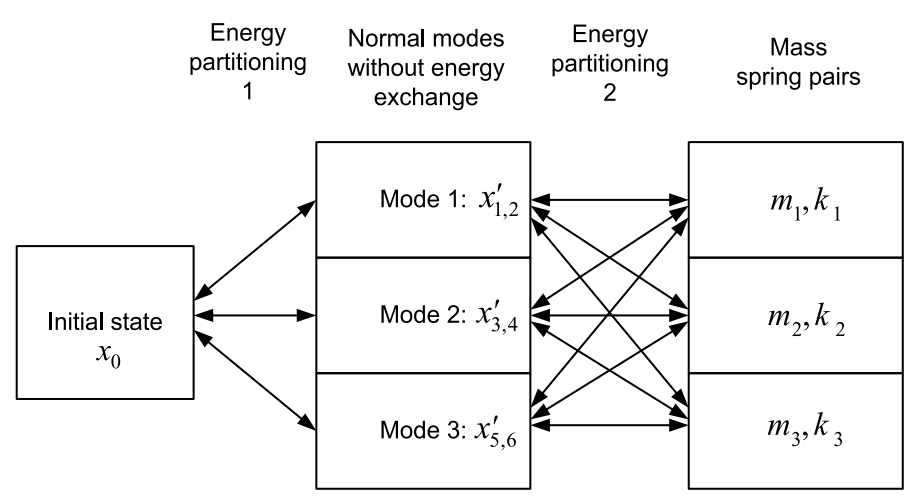

Fig. 2 Fixed (1) and time-varying and (2) energy partitioning in a linear mass-spring system

Equipartition of energy happens if and only if in Fig. 1 the energy partitioning 2 associated to each normal mode is an equipartition over all 3 mass-spring pairs $m_{i}, k_{i}$, $i=1,2,3$.

There is a subtle but important difference between the energy partitionings 1 and 2 in Fig. 2. Energy partitioning 1 is fixed and applies at time zero whereas energy partitioning 2 applies at any time, is time-varying (oscillatory), and may even be negative. At any time however the sum of the 3 components connected to each normal mode is fixed to the nonnegative value determined by the initial condition. Similarly the sum of the 3 components connected to each mass-spring pair is nonnegative. Equipartition relates to the time-average in the limit as time goes to infinity of all 9 components represented by the 9 arrows on the right in Fig. 2.

\section{A Test for Energy Equipartition}

The normal modes of a linear diagonalizable conservative system as represented by (4.7), (4.5) do not interact. This implies (4.12), (4.14), (4.15). Assuming (4.16) this also implies (4.17). These relations offer a simple efficient way to compute whether two energies equipartition.

Consider a linear diagonalizable conservative system (2.2). Energies present in this system are all represented by quadratic expressions of the form

$$
x^{\mathrm{T}}(t) S x(t)
$$

where $S$ is nonnegative symmetric. Two arbitrary energies $E_{k}, k=1,2$, associated to the system (2.2) are therefore represented by,

$$
E_{k}(t)=x^{\mathrm{T}}(t) S_{k} x(t), \quad k=1,2
$$

where $S_{k}, k=1,2$, are nonnegative symmetric matrices that determine the energies. For $E_{k}(t), k=1,2$, to equipartition necessary and sufficient conditions concerning 
$S_{k}, k=1,2$, will be established. Apply to the system (2.2) the basis transformation (4.6). Then the normal mode representation (4.7), (4.5) is obtained. The energies (5.1) in the normal mode representation are

$$
E_{k}(t)=x^{\prime \mathrm{T}}(t) S_{k}^{\prime} x^{\prime}(t), \quad S_{k}^{\prime}=V^{\prime \mathrm{T}} S_{k} V^{\prime}, k=1,2
$$

with $S_{k}^{\prime}, k=1,2$, nonnegative symmetric. According to (3.5), (3.6) the two energies $E_{k}(t), k=1,2$, equipartition if

$$
\forall x^{\prime}(0): \quad \bar{E}_{1}=\lim _{T \rightarrow \infty} \frac{1}{T} \int_{0}^{T} x^{\prime \mathrm{T}}(t) S_{1}^{\prime} x^{\prime}(t) d t=\bar{E}_{2}=\lim _{T \rightarrow \infty} \frac{1}{T} \int_{0}^{T} x^{\prime \mathrm{T}}(t) S_{2}^{\prime} x^{\prime}(t) d t
$$

The time-averaged energies $\bar{E}_{k}, k=1,2$, can be expressed as follows:

$$
\begin{aligned}
\bar{E}_{k} & =\sum_{j=1,3,5, \ldots}^{2 n-1} \lim _{T \rightarrow \infty} \frac{1}{T} \int_{0}^{T}\left[x_{j}^{\prime}(t) x_{j+1}^{\prime}(t)\right]\left[\begin{array}{cc}
S_{k_{j, j}}^{\prime} & S_{k_{j, j+1}}^{\prime} \\
S_{k_{j+1, j}}^{\prime} & S_{k_{j+1, j+1}}^{\prime}
\end{array}\right]\left[\begin{array}{c}
x_{j}^{\prime}(t) \\
x_{j+1}^{\prime}(t)
\end{array}\right] d t, \\
k & =1,2
\end{aligned}
$$

Using the normal mode properties (4.14), (4.15), (4.17) from (5.5) we obtain

$$
\bar{E}_{k}=\sum_{j=1,3,5, \ldots}^{2 n-1}\left(S_{k_{j, j}}^{\prime}+S_{k_{j+1, j+1}}^{\prime}\right)\left(x_{j}^{\prime 2}(0)+x_{j+1}^{\prime 2}(0)\right), \quad k=1,2
$$

$E_{k}(t), k=1,2$, equipartition if $\bar{E}_{k}, k=1,2$, are equal for every $x^{\prime}(0)$. From (5.6) the following conditions are therefore necessary and sufficient for $E_{k}(t), k=1,2$, to equipartition:

$$
S_{1_{j, j}}^{\prime}+S_{1_{j+1, j+1}}^{\prime}=S_{2_{j, j}}^{\prime}+S_{2_{j+1, j+1}}^{\prime}, \quad j=2(i-1)+1, i=1,2, \ldots, n, k=1,2
$$

Equation (5.7) states that all corresponding $2 \times 2$ matrices that appear in (5.5) should have identical traces. Equation (5.6) states that time-average energy $\bar{E}_{k}$ depends in a simple algebraic manner on the trace of $S_{k}^{\prime}$ and the initial conditions $x^{\prime}(0)$. This relation provides highly valuable insight in energy equipartition and constitutes a highly efficient way to compute time-averaged energies for the system (2.2). It is obtained from a normal mode representation of the system.

Example 1 Confirmation of the virial theorem. The virial theorem states that potential and kinetic energy in any conservative system equipartition. For the mass-spring system on top of Fig. 1 we verify this by checking whether the necessary and sufficient condition (5.7) for equipartition is satisfied. The values of the masses and spring constants are selected randomly,

$$
\begin{array}{llll}
m_{1}=1.4501, & m_{2}=0.7311, & m_{3}=1.1068, & m_{4}=0.9860 \\
k_{1}=1.3913, & k_{2}=1.2621, & k_{3}=0.9565, & k_{4}=0.5185
\end{array}
$$


Let $x^{\mathrm{T}}(t) S_{1} x(t)$ and $x^{\mathrm{T}}(t) S_{2} x(t)$ represent the potential and kinetic energy of the system respectively. Then,

$$
\begin{gathered}
S_{1}=K_{s}^{\mathrm{T}} K_{s}, \quad K_{s}=\left[\begin{array}{cccc}
-1.1795 & 0 & 0 & 0 \\
1.1234 & -1.1234 & 0 & 0 \\
0 & 0.9780 & -0.9780 & 0 \\
0 & 0 & 0.7201 & -0.7201
\end{array}\right] \\
S_{2}=\operatorname{diag}\left(\frac{1}{m_{1}}, \frac{1}{m_{2}}, \frac{1}{m_{3}}, \frac{1}{m_{n}}\right)=\operatorname{diag}\left(\frac{1}{1.4501}, \frac{1}{0.7311}, \frac{1}{1.1068}, \frac{1}{0.9860}\right)
\end{gathered}
$$

Transformation to normal mode representation of $S_{1}, S_{2}$ according to (5.3) gives:

$$
\begin{aligned}
& S_{1}^{\prime}=V^{\prime \mathrm{T}} S_{1} V^{\prime}=\operatorname{diag}(00.680400 .76580 .452600 .12100) \\
& S_{2}^{\prime}=V^{\prime \mathrm{T}} S_{2} V^{\prime}=\operatorname{diag}(0.680400 .7658000 .452600 .1210)
\end{aligned}
$$

From (5.11) observe that the necessary and sufficient condition (5.7) for equipartition is satisfied.

\section{Total Equipartition}

The second law of thermodynamics applies only on a macroscopic space and time scale. The mass-spring systems considered in this paper are meant to represent the micro-scale of the system. Therefore to investigate the second law we have to consider mass-spring systems with a large enough number of masses and springs. The second law of thermodynamics when applied to systems with identical components on the micro-scale states that energy equipartition is achieved regardless of the initial condition. Equipartition in this case applies to identical parts of the system that are large enough. According to (3.6) equipartition concerns time-averaged energies and therefore automatically considers a large enough time-scale.

In the next section we will consider equipartition of energy of mass-spring systems separated into equal parts that are large enough (macroscopic). In this section equipartition of energy on arbitrary scales is considered. The result can be used to investigate equipartition of energy on both macroscopic and microscopic scales. Although equipartition on a microscopic scale is seldom achieved in practice, it is a sufficient condition for equipartition on a larger scale.

Consider the linear diagonalizable conservative system (2.2) with $n$ normal modes and distinct eigenvalues. For this system consider $m \geq 2$ energies in normal mode representation,

$$
x^{\prime \mathrm{T}}(t) S_{k}^{\prime} x^{\prime}(t), \quad k=1,2, \ldots, m
$$

According to (5.7) all these energies equipartition if

$$
\begin{aligned}
& S_{k_{j, j}}^{\prime}+S_{k_{j+1, j+1}}^{\prime}=S_{l_{j, j}}^{\prime}+S_{l_{j+1, j+1}}^{\prime} \\
& \quad j=2(i-1)+1, i=1,2, \ldots, n, k, l \in\{1,2, \ldots, m\}
\end{aligned}
$$


The energies (6.1) make up the total energy of the system if

$$
\forall x^{\prime}: \quad \sum_{k=1}^{m} x^{\prime \mathrm{T}}(t) S_{k}^{\prime} x^{\prime}(t)=x^{\prime \mathrm{T}}(t) Q^{\prime} x^{\prime}(t)
$$

When the energies (6.1) satisfy (6.2), (6.3) this is called total equipartition. Equation (6.3) is equivalent with

$$
\sum_{k=1}^{m} S_{k}^{\prime}=Q^{\prime}
$$

Since $Q^{\prime}$ is diagonal (6.4) translates into,

$$
\begin{gathered}
\sum_{k=1}^{m} S_{k_{j, j}}^{\prime}=Q_{j, j}^{\prime}, \quad j=1,2, \ldots, 2 n \\
\sum_{k=1}^{m} S_{k_{i, j}}^{\prime}=0, \quad i \neq j, i, j \in\{1,2, \ldots, 2 n\}
\end{gathered}
$$

Using (6.5), (6.2) becomes

$$
S_{k_{j, j}}^{\prime}+S_{k_{j+1, j+1}}^{\prime}=\frac{1}{m}\left(Q_{j, j}^{\prime}+Q_{j+1, j+1}^{\prime}\right), \quad j=2(i-1)+1, k=1,2, \ldots, m
$$

In summary for total equipartition the matrices $S_{k}^{\prime}$ in (6.1) have to satisfy (6.5)-(6.7) while they must also come out nonnegative symmetric.

To interpret the conditions (6.5)-(6.7) use (4.10), (6.7) to find that

$$
\begin{gathered}
{\left[\begin{array}{ll}
x_{j}^{\prime}(t) & x_{j+1}^{\prime}(t)
\end{array}\right]\left[\begin{array}{cc}
S_{k_{j, j}^{\prime}} & 0 \\
0 & S_{k_{j+1, j+1}}^{\prime}
\end{array}\right]\left[\begin{array}{c}
x_{j}^{\prime}(t) \\
x_{j+1}^{\prime}(t)
\end{array}\right]=\frac{1}{m} E_{i}^{m},} \\
j=2(i-1)+1, \quad i=1,2, \ldots, n, \quad k=1,2, \ldots, m
\end{gathered}
$$

and so,

$$
x^{\prime \mathrm{T}}(t) \operatorname{diag}\left(S_{k}^{\prime}\right) x^{\prime}(t)=\frac{1}{m} \sum_{i=1}^{n} E_{i}^{m}=\frac{H}{m}, \quad k=1,2, \ldots, m
$$

where $H$ denotes the total energy in the system. According to (6.9) the matrices $\operatorname{diag}\left(S_{k}^{\prime}\right), k=1,2, \ldots, m$ already achieve the desired equipartition of energy. This must imply that the off-diagonal elements of $S_{k}^{\prime}, k=1,2, \ldots, m$, when they satisfy (6.6), cause a redistribution of energy at all times the time-average of which is zero.

Combining equations (6.8), (6.7), (4.11) gives the following interpretation. For the total equipartition of energy, $S_{k}^{\prime}, k=1,2, \ldots, m$ should be such that they take an equal $1 / m$ portion of the energy $E_{i}^{m}=E_{i}^{m p}+E_{i}^{m k}$ of each normal mode $i=$ $1,2, \ldots, n$. We may select $S_{k_{j, j}}^{\prime}=Q_{j, j}^{\prime} / m, S_{k_{j+1, j+1}^{\prime}}^{\prime}=Q_{j+1, j+1}^{\prime} / m$ to satisfy (6.7). This means selecting an equal $1 / m$ portion of both $E_{i}^{m p}$ and $E_{i}^{m k}$. According to (6.7) 
however we may also take e.g. a larger portion of $E_{i}^{m p}$ and a smaller portion of $E_{i}^{m k}$ as long as their sum equals the $1 / m$ portion of $E_{i}^{m}$. But (6.5) does require that the sum over all normal modes of these possibly larger and smaller portions of $E_{i}^{m p}$ and $E_{i}^{m k}$, $i=1,2, \ldots, n$, respectively, add up to the total potential and total kinetic energy in the system at any time.

Summarizing ones more. The diagonal elements of $S_{k}^{\prime}, k=1,2, \ldots, m$, have to satisfy (6.5), (6.7). Equation (6.7) ensures equipartioning while (6.5) ensures that the energies together are equal to the total constant energy of the system at all times. The off-diagonal elements of $S_{k}^{\prime}, k=1,2, \ldots, m$, may be set to zero since these only cause a redistribution of energy at all times the time-average of which is zero. If taken non-zero they must satisfy (6.6) and be such that $S_{k}^{\prime}, k=1,2, \ldots, m$, all come out nonnegative symmetric.

Example 2 Conjecture Bhat and Bernstein [4]. Consider the linear conservative system:

$$
\begin{gathered}
\dot{x}(t)=A x(t), \quad A=\left[\begin{array}{ccccc}
\hat{A} & \hat{C} & \hat{C} & \ldots & \hat{C} \\
-\hat{C}^{\mathrm{T}} & \hat{A} & \hat{C} & \ldots & \hat{C} \\
-\hat{C}^{\mathrm{T}} & -\hat{C}^{\mathrm{T}} & \hat{A} & \ldots & \hat{C} \\
\vdots & \vdots & \vdots & & \vdots \\
-\hat{C}^{\mathrm{T}} & -\hat{C}^{\mathrm{T}} & -\hat{C}^{\mathrm{T}} & \ldots & \hat{A}
\end{array}\right] \in R^{2 n \times 2 n}, \\
\hat{A}=\left[\begin{array}{cc}
0 & \omega \\
-\omega & 0
\end{array}\right], \quad \omega>0, \quad \hat{C}=\left[\begin{array}{ll}
\hat{a} & \hat{b} \\
\hat{c} & \hat{d}
\end{array}\right], \quad \hat{c}=\hat{b}
\end{gathered}
$$

Observe that the system description matches the one given by Bhat and Bernstein. We have added ourselves the condition $\hat{c}=\hat{b}$ i.e. the condition that $\hat{C}$ is symmetric. Bhat and Bernstein considered the following energies associated to the system (6.10),

$$
E_{i}(t)=\frac{1}{2} x_{2 i-1}^{2}(t)+\frac{1}{2} x_{2 i}^{2}(t), \quad i=1,2, \ldots, n
$$

where $\sum_{i=1}^{n} E_{i}(t)$ is the total constant energy $H$ in the system. Their conjecture states total equipartition of the energies (6.11). To investigate their conjecture we used random values for $\omega>0, \hat{a}, \hat{b}, \hat{d}$ and several values for $n$. To check the equipartition observe from (6.11),

$$
E_{i}(t)=x^{\mathrm{T}}(t) S_{i}(t) x(t), \quad i=1,2, \ldots, n
$$

where $S_{i}$ is a diagonal matrix with all diagonal elements equal to zero except for elements $2 i-1,2 i-1$ and $2 i, 2 i$ that are equal to 1 . According to (5.3) the normal mode representation of the matrices $S_{i}, i=1,2, \ldots, n$, are

$$
S_{i}^{\prime}=V^{\prime \mathrm{T}} S_{i} V^{\prime}, \quad i=1,2, \ldots, n
$$

Because the energies (6.11) add up to the total energy in the system, for total equipartition it suffices to check the conditions (6.2) that are similar to (5.7). Note that the 
index $k$ in (6.2) corresponds to the index $i$ in (6.13) and that $m$ in (6.2) equals $n$. The many different examples we tried all confirmed the conjecture, as long as $\hat{C}$ is symmetric. All examples we tried with an asymmetric $\hat{C}$ failed the conjecture. As just one example we state the result for $n=3, \omega=0.6059, \hat{a}=2.1458, \hat{b}=0.3440$, $\hat{d}=2.9688$. The conditions (6.2) only involve the diagonal elements of $S_{i}^{\prime}, i=1,2,3$. These are as follows:

$$
\begin{array}{cc}
S_{1}^{\prime}: & 0.2860,0.0473,0.2860,0.0473,0.1667,0.1667 \\
S_{2}^{\prime}: & 0.1363,0.1970,0.0777,0.2556,0.1667,0.1667 \\
S_{3}^{\prime}: & 0.0777,0.2556,0.1363,0.1970,0.1667,0.1667
\end{array}
$$

The diagonal elements in (6.14) are rounded up to 4 decimals but the conditions (6.2) are all satisfied close to the machine precision.

Although the paper by Bernstein and Bhat suggests that (6.10) is a representation of a mass-spring system we encountered difficulties in obtaining its physical mass-spring realisation. Although total energy equipartition is achieved for the system (6.10) we were unable to find a mass-spring system obeying total equipartition taking as the system parts mass-spring pairs that are physically connected. This suggests that total equipartition rarely occurs.

\section{Emergence of the Second Law}

Total equipartition is only achieved if the system dynamics meet highly special demands. Yet the second law of thermodynamics, that is believed to be universal, seems to imply total equipartition. This appears to be a contradiction. The second law of thermodynamics however applies to macroscopic space and time scales, not microscopic ones. Therefore the second law is an emergent property. To resolve the apparent contradiction recall that the second law only demands that equipartition of energy is approximated in the limit as the space and time scale increase. The approximation of equipartition of energy is reflected by the fact that the necessary and sufficient condition (5.7) for total equipartition will not be satisfied while on the other hand the time-averaged energies (5.6) of the system parts $k=1,2, \ldots, N$ will become equal in the limit $n \rightarrow \infty$, regardless of the initial conditions.

To demonstrate that the second law of thermodynamics is an emergent property in this section the ectropy (3.7) is computed as a function of the size of identical system parts of a mass-spring system. Recall that ectropy as defined by (3.7) is a measure of how far the system is removed from equipartition of energy. In this section this will be interpreted as how far the system is removed from satisfying the second law of thermodynamics. It will be shown in this section that as the size of system parts grows ectropy tends to zero regardless of the initial conditions. This confirms the emergent character of the second law.

The mass-spring system at the top of Fig. 1 will be considered, extended on the right with arbitrarily many springs and masses. All masses $m_{i}, i=1,2, \ldots, n$, have identical values equal to one and the spring constants $k_{i}, i=1,2, \ldots, n$, associated to the springs have also identical values equal to one. The system will 
be separated into $N$ identical physically connected parts starting from the left in Fig. 1 where $N$ is a divisor of $n$. So part $i=1,2, \ldots, N$ consists of masses $m_{(i-1) n / N+1}, m_{(i-1) n / N+2}, \ldots, m_{i n / N}$ and springs $s_{(i-1) n / N+1}, s_{(i-1) n / N+2}, \ldots$, $s_{i n / N}$. For different values of $n, N$, Fig. 3 records the average and maximum ectropy $U$, given by (3.7), obtained from $n$ different initial conditions,

$$
x(0)=\left[\begin{array}{llllllll}
p_{1}(0) & p_{2}(0) & \ldots & p_{n}(0) & \dot{p}_{1}(0) & \dot{p}_{2}(0) & \ldots & \dot{p}_{n}(0)
\end{array}\right]^{\mathrm{T}}
$$

Using $n$ different initial conditions (7.1), and plotting the average and maximum (worst case) ectropy $U$ obtained from them, is meant to show that equipartition of energy is approached regardless of the initial conditions. The $n$ different initial conditions are computed as follows:

$$
\begin{aligned}
& x_{r}(0)=\left[\begin{array}{llllllll}
1 * r_{1} & 2 * r_{2} & \ldots & n * r_{n} & 1 * r_{n+1} & 2 * r_{n+2} & \ldots & n * r_{2 n}
\end{array}\right]^{\mathrm{T}} \text {, } \\
& x(0)=\frac{100}{x_{r}^{\mathrm{T}}(0) Q_{r} x_{r}(0)} x_{r}(0)
\end{aligned}
$$

In (7.2) $r_{j}, j=1,2, \ldots, 2 n$, are normally distributed zero mean random numbers with variance one. Their distribution can be written as $N(0,1)$. Furthermore $x_{r}^{\mathrm{T}}(0) Q_{r} x_{r}(0)$ represents the total energy associated to the initial state $x_{r}(0)$ that is used to normalize the initial state $x(0)$ so that the total energy in each system equals 100 , regardless of its state dimension $2 n$. This is necessary to enable a fair comparison of the ectropy values as defined by (3.7) because these increase with the total energy present in the system. By multiplying the random numbers in $x_{r}(0)$ in the manner (7.2) a high initial value $U(0)$ of the ectropy (3.4) is achieved because the initial energy in each mass and spring increases on average as we move from left to right at the top in Fig. 1. The high initial value $U(0)$ is used to ensure that the initial condition is far from energy equipartition.

To compute the ectropy $U$ in (3.7) the time-averaged energies $\bar{E}_{k}$ of each system part $k=1,2, \ldots, N$ need to be computed. For system part $k=1,2, \ldots, N$, similar to (5.2), we first determine the nonnegative symmetric matrix $S_{k}$ so that $E_{k}(t)=x^{\mathrm{T}}(t) S_{k} x(t)$ represents the energy of this system part at each time $t$. To do this the rules mentioned below equation (2.2) are used. Next the nonnegative symmetric matrices $S_{k}^{\prime}, k=1,2, \ldots, N$, associated to the normal mode representation are computed using equations (4.1)-(4.3), (5.3). Then, using (5.6), the time-averaged energies $\bar{E}_{k}, k=1,2, \ldots, N$, are computed.

From the left Fig. 3 observe that as the number of masses and springs $n / N$ of the $N=10$ identical system parts grows both the average and maximum ectropy decrease towards zero meaning that energy equipartition is approached. The maximum values represent the trend of the worst (highest) values of the ectropy $U$ whereas the average represents the trend of the average ectropy $U$ for increasing system dimensions. Due to the partly random nature of the $n$ initial conditions (7.2), as expected, the decrease of the maximum (worst case) ectropy is less smooth and monotonic. From the right Fig. 3 a similar conclusion may be drawn. Only now instead of the number of system parts $N$ the systems total number of masses and springs $n=1000$ is fixed. As announced at the start of this section Fig. 3 confirms the emergent character of the 

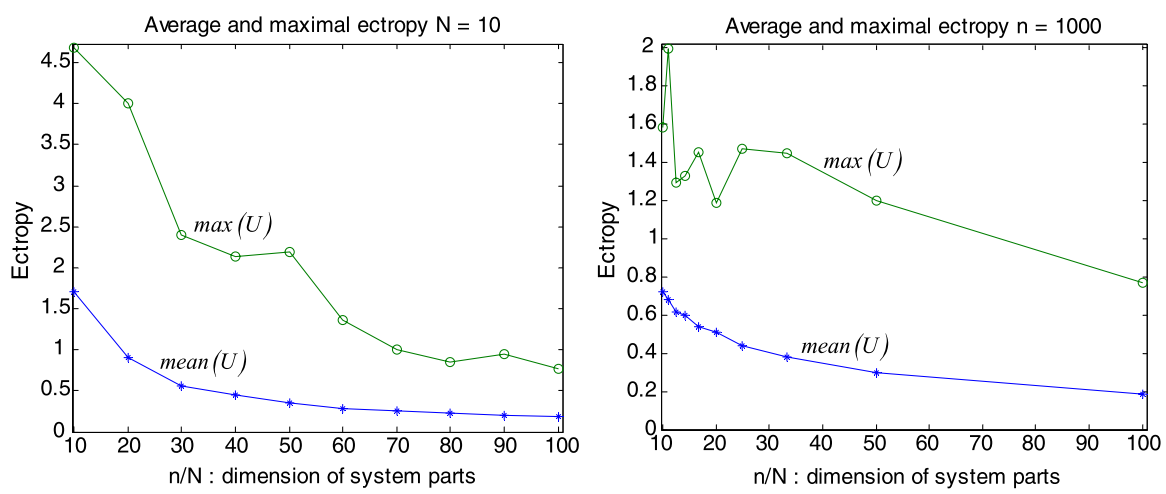

Fig. 3 Decrease of the average ectropy mean $(U)$ and worst case ectropy $\max (U)$ with increasing size $n / N$ of system parts for fixed values $N=10$ (left) and $n=1000$ (right)

second law because the ectropy, that measures the distance from energy equipartition i.e. satisfaction of the second law, goes to zero as the size of the system parts grows.

To show that equipartition of energy occurs regardless of the initial conditions the computations associated to Fig. 3 were performed for different initial conditions (7.1), (7.2). Although this might suggest invoking some kind of statistical mechanics observe that no probabilistic computations, probabilistic descriptions or probabilistic measures are used by us to arrive at Fig. 3. Neither have we used the ergodic hypothesis concerning the equality of time and ensemble averages or equilibrium assumptions. The only thing we have exploited are the simple reversible deterministic dynamics of a large collection of masses and springs and their dynamic responses.

For one-dimensional mass-spring systems with equal masses and springs Fig. 3 proofs that the second law of thermodynamics is an emergent property. To prove an emergent property one must show that it is satisfied more closely as the dimension of the system increases. This in turn requires a measure of how closely the property is satisfied. The measure we used is the ectropy (3.7) and Fig. 3 shows how it tends to zero as the system dimension increases. This implies that the second law of thermodynamics is more closely satisfied as the system dimension increases while in the limit energy equipartition occurs.

If the partitioning of the system is a partitioning in parts $k=1,2, \ldots, N$ that are identical in size, as they were in Fig. 3, the second law comes down to energy equipartition. But the second law is actually about the equipartition of temperature. To make the transition from equipartition of energy to equipartition of temperature thermal capacities $h_{k}$ must be attached to the (macroscopic) system parts $k=1,2, \ldots, N$ [2]. The thermal capacities provide the relation between energy and temperature:

$$
T_{k}(t)=\frac{1}{h_{k}} E_{k}(t), \quad k=1,2, \ldots, N
$$

If we take system parts that are unequal in size the second law instead of equipartition of energy demands equipartition of temperature. As an example we considered the same system as before with a total number of masses and springs equal to $n$. This 
system was divided into unequal parts with $n_{k}, k=1,2, \ldots, N$, consecutive springs and masses such that,

$$
\sum_{k=1}^{N} n_{k}=n
$$

We selected:

$$
\begin{aligned}
& n=1000, \quad N=10, \quad n_{k}=50+10 k, \quad k=1,2,3,4,5, \\
& n_{k}=100+10(k-6), \quad k=6,7,8,9,10
\end{aligned}
$$

The thermal capacities associated to the system parts must be proportional to the size of the system parts. We selected

$$
h_{k}=\frac{N}{n} n_{k}
$$

The ectropy $U_{T}$, specified below, is similar to the ectropy $U$ in (3.7). The difference is that $U$ measures how far the system is removed from energy equipartition whereas $U_{T}$ measures how far the system is removed from temperature equipartition,

$$
U_{T}=\sum_{k=1}^{N}\left|\bar{T}_{k}-\bar{T}\right|, \quad \bar{T}=\frac{1}{N} \sum_{k=1}^{N} T_{k}, \quad \bar{T}_{k}=\lim _{T \rightarrow \infty} \frac{1}{T} \int_{0}^{T} T_{k}(t) d t
$$

Using (7.3), (7.6) the computation of $U$ in (3.7) is easily extended to that of $U_{T}$ in (7.7). Observe that if the energy totally equipartitions over the $n$ identical (microscopic) mass-spring pairs then according to (7.3), (7.6), (7.7) this implies both temperature equipartition,

$$
T_{1}=T_{2}=\cdots=T_{N} \quad \Leftrightarrow \quad U_{T}=0
$$

and

$$
H=\sum_{k=1}^{N} T_{k}=N T_{1}=N T_{2}=\cdots=N T_{N}
$$

The average value of $U_{T}$ for the system characterized by (7.5), (7.6), obtained from $n=1000$ initial conditions (7.2), was computed to be 0.1958 . The maximum value of $U_{T}$ was 0.7931 . These values may be compared with the terminal values in Fig. 3 and indicate the emergence of temperature equipartition. This comparison is justified because of equation (7.9) that holds for total energy equipartition over the $n$ identical (microscopic) mass-spring pairs.

\section{Ectropy Decrease and Loschmidt's Paradox}

The second law of thermodynamics not only predicts temperature equipartition, as described and illustrated in the previous section, but also states that the ectropy $U(t)$, given by (3.4), decreases on a large enough time-scale. The left Fig. 4 shows 3 simulations of the ectropy $U(t)$ with initial conditions, given by (7.2), having a high ectropy 

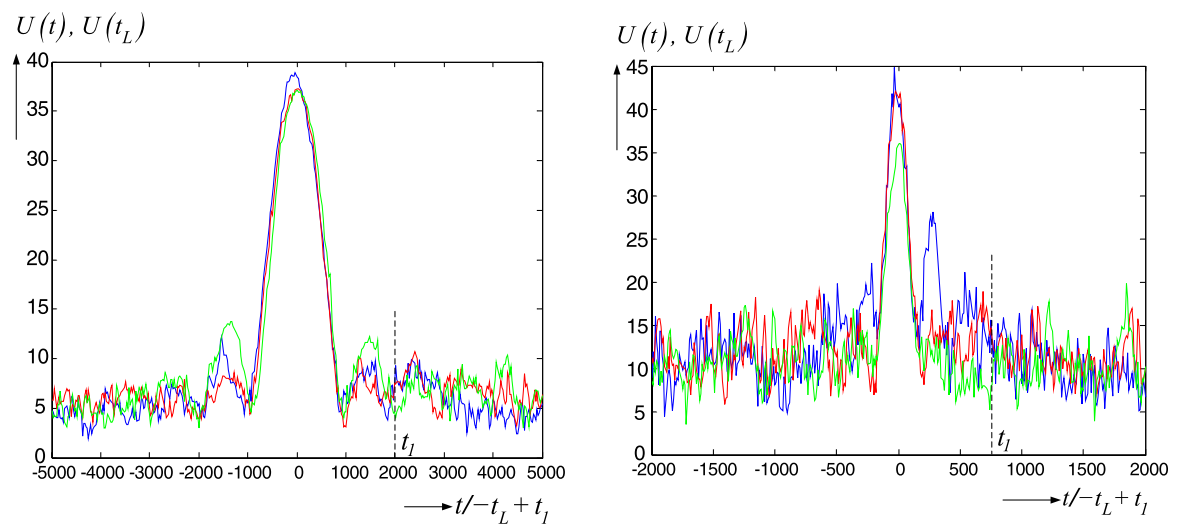

Fig. 4 Ectropy responses starting from high ectropy levels at time $t=0$. Left: $n=1000, N=10,3$ responses. Right: $n=200, N=10,3$ responses

level. The system is the mass-spring system from the previous section with $n=1000$ and $N=10$. The ectropy $U(t)$, given by (3.4), is easily computed for arbitrary times $t$ from (4.12), (4.13), (5.5). These equations enable an efficient computation of ectropy over arbitrary time intervals. We actually performed many more simulations of the type shown in Fig. 4. They all show the same pattern. Starting from a high value the ectropy, apart from fluctuations, significantly decreases after some time and finally starts to fluctuate on an ectropy level higher than zero. The fluctuations are due to permanent exchanges of energy at the micro-scale that do not perfectly cancel out. In the system (2.2) these are energy exchanges between the individual masses and springs. Fluctuations of ectropy (entropy) on the micro-scale have been confirmed experimentally [8]. These fluctuations decrease as the macroscopic dimensions increase. To demonstrate this the right Fig. 4 shows the same simulations but with the macroscopic dimensions reduced with a factor $5(n=200, N=10)$. Observe that the fluctuations increase. The consistent significant decrease of ectropy in Fig. 4 confirms the decrease of ectropy stated by the second law on a large enough time-scale where fluctuations are negligible.

Our attempt to explain the second law of thermodynamics from just the simple reversible dynamic laws that govern motion and energy at the micro-scale faces Loschmidt's paradox. In terms of ectropy simulations, this paradox can be stated as follows. Simulations starting from a high level of ectropy at time $t=0$, apart from fluctuations, consistently show a decrease of ectropy as illustrated by Fig. 4. At any time $t>0$ during the simulation we may reverse the sign of the velocities $\dot{p}_{i}(t)$, $i=1,2, \ldots, n$, of the state $x(t)$ given by (2.1) to obtain a new state

$$
x_{r}(t)=\left[\begin{array}{llllll}
p_{1}(t) & \ldots & p_{n}(t) & -\dot{p}_{1}(t) & \ldots & -\dot{p}_{n}(t)
\end{array}\right]^{\mathrm{T}} \in R^{2 n}
$$

If we perform a new simulation the state and time of which are denoted by $x_{L}, t_{L}$ and we take as the new initial state

$$
x_{L}\left(t_{L}=0\right)=x_{r}\left(t=t_{1}\right), \quad t_{1}>0
$$


the state provided by this new simulation will satisfy

$$
x_{L}\left(t_{L}\right)=x_{r}\left(t=t_{1}-t_{L}\right), \quad 0 \leq t_{L} \leq t_{1}
$$

In other words the new state $x_{L}\left(t_{L}\right), 0 \leq t_{L} \leq t_{1}$, will travel the states $x_{r}(t), 0 \leq$ $t \leq t_{1}$, in time reversed order. This property is due to the reversible (Hamiltonian) nature of the dynamic equations of the system at the micro-scale [9]. From (2.1), (8.1) observe that $x(t)$ and $x_{r}(t)$ give identical values for the ectropy $U(t)$, given by (3.4), because the sign change of the velocities leaves all kinetic energies unchanged. Therefore we must conclude that

$$
U\left(t_{L}\right)=U\left(t=t_{1}-t_{L}\right), \quad 0 \leq t_{L} \leq t_{1}
$$

so the ectropy values are traveled in time-reversed order too. Therefore $x_{L}\left(t_{L}\right), U\left(t_{L}\right)$, $0 \leq t_{L} \leq t_{1}$ is a simulation of the system (2.2) that shows a significant increase in ectropy on a macroscopic time and space scale. This seems inconsistent with the second law.

What may be concluded from the simulations described above? Observe that the initial conditions (7.2) are all roughly on the same high ectropy level. Apart from this level they are selected randomly. By taking these "arbitrary initial conditions with a high almost equal ectropy level" simulations consistently confirm the decrease of ectropy consistent with the second law. On the other hand any initial condition constructed according to (8.2) results in a significant ectropy increase that seems to refute the second law. The initial states (8.2) can be constructed in an infinite number of ways. Therefore one might expect to also encounter an increase of ectropy in simulations starting from arbitrary initial conditions. To investigate this we set up a computer experiment where we performed many simulations the initial states of which were selected similar to (7.2),

$$
\begin{aligned}
& x_{r}(0)=\left[\begin{array}{llllll}
1^{c} * r_{1} & 2^{c} * r_{2} & 3^{c} * r_{3} & \ldots & n^{c} * r_{n} \\
& 1^{c} * r_{n+1} & 2^{c} * r_{n+2} & 3^{c} * r_{n+3} & \ldots & n^{c} * r_{2 n}
\end{array}\right]^{\mathrm{T}}, \\
& x(0)=\frac{100}{x_{r}^{\mathrm{T}}(0) Q_{r} x_{r}(0)} x_{r}(0)
\end{aligned}
$$

By varying the constant $c, 0 \leq c \leq 1$, in (8.5) the initial value of ectropy is manipulated. The lowest value is obtained for $c=0$ and the highest, which is equal to the one produced by (7.2), is obtained for $c=1$. Apart from the initial ectropy value the selection of the initial state (8.5) is random. The computer experiment searched for a significant ectropy increase that exceeds the level of fluctuations. The procedure to detect these was verified using initial states (8.2). For each value $c=0,0.1,0.2, \ldots, 1$ in (8.5) the computer experiment performed 1000 simulations of the system considered in the previous section with $n=1000$ and $N=10$. A significant increase of ectropy was never found on the time-scale of the simulation $(0 \leq t \leq 5000)$. This must mean that although infinitely many initial states exist that lead to a significant ectropy increase at the time-scale of the simulations, together these initial states form a negligible part within sets of initial states in a certain small ectropy range. So 
we may conclude from our computer experiment that the initial states constructed according to (8.2) are highly unlikely. This implies that the second law of thermodynamics should be rephrased as stating that the decrease of ectropy occurs with high probability. As argued in the next paragraph, from the point of view of a physicist, the states (8.2) never occur and the ectropy $U(t)$, given by (3.4), always decreases.

Loschmidt's paradox is strongly associated with what is called the Poincaré recurrence property of conservative Hamiltonian systems. This property states that conservative Hamiltonian systems return very close to their initial state infinitely often. Since ectropy is a function of the state this implies that ectropy behaves cyclic. The time of a cycle however is known to increase dramatically with the dimension of the system. For an ideal gas observed at the macroscopic human scale it has been calculated to exceed by orders of magnitude the time we believe the universe exists since the big-bang. Then we may ask: "Is Poincaré recurrence incompatible with the second law of thermodynamics?" Hans Christian von Bayer [10] (page 141) stated the answer very nicely: "In dealing with outrageously large numbers mathematicians and physicists part company".

Poincaré recurrence offers a way to interpret the constructed initial states (8.2) that are highly unlikely (never) to occur. Although the recurrence time is exceptionally long, in simulation nothing prevents us from considering the time and state the cycle completes. This time and state are represented by $t_{L}=t_{1}$ and $x\left(t_{L}=t_{1}\right)$ in (8.2). Starting from just before this end of the cycle, i.e. from $t_{L}=0$ and $U\left(t_{L}=0\right)$, ectropy increases significantly back to its initial value $U\left(t_{L}=t_{1}\right)$ as shown in Fig. 4 . The fact that at $t_{L}=t_{1}$ the cycle is completed is confirmed by simulating $x\left(t_{L}\right), U\left(t_{L}\right)$ for $t_{L}>t_{1}$ because the ectropy $U\left(t_{L}\right)$ starts to decrease for $t_{L}>t_{1}$ in a similar manner as observed for $t>0$ in Fig. 4.

In summary simulations starting at $t=0$ from initial states $x(t=0)$ with a certain ectropy $U(x(t=0))$ but otherwise random, apart from fluctuations, consistently show a decrease of ectropy: $U\left(x\left(t=t_{1}\right)\right)<U(x(t=0)), t_{1}>0$. At any moment in time $t=t_{1}>0$ we may reverse all the velocities of the state $x\left(t=t_{1}\right)$ to obtain a new state $x_{r}\left(t=t_{1}\right)$ given by (8.1) with ectropy $U\left(x_{r}\left(t=t_{1}\right)\right)=U\left(x\left(t=t_{1}\right)\right)<$ $U(x(t=0))$. If we simulate starting from $t_{L}=0$ with initial state $x_{r}\left(t_{L}=0\right)=$ $x_{r}\left(t=t_{1}\right)$ all these simulations will violate the second law i.e. $U\left(x_{r}\left(t_{L}=t_{1}\right)\right)=$ $U(x(t=0))>U\left(x_{r}\left(t_{L}=0\right)\right), t_{1}>0$. However within the set of all states having an ectropy equal to $U\left(x_{r}\left(t_{L}=0\right)\right)$ the states $x_{r}\left(t_{L}=0\right)=x_{r}\left(t=t_{1}\right)$ constructed from simulations that started from a higher ectropy $U(x(t=0))$ form a negligible part.

\section{Conclusions, Interpretations and Speculations}

Using just the simple, reversible dynamic laws of masses and springs governing the systems micro-scale we demonstrated how the second law of thermodynamics emerges from them. The second law of thermodynamics implies temperature equipartition but only on a large enough space and time scale, not on a smaller (micro) scale. This was shown by means of dynamic simulations and efficient computations of timeaveraged energies of conservative linear large scale dynamical systems. In addition the dual notion of entropy called ectropy was employed. We modified ectropy such 
that its minimum is zero and its unit becomes that of energy. This modification allows us to interpret ectropy, as given by (3.4), as usable energy (macroscopic energy) if all macroscopic system parts are (approximately) identical. Zero ectropy then describes energy equipartition among all macroscopic system parts. After reaching zero ectropy macroscopic energy transfer is no longer possible. The extension to equipartition of temperature that applies to unequal macroscopic system parts was made through the introduction of thermal capacities.

In the past many attempts have been made to seek confirmation of the second law by means of simulations of systems governed by just the simple reversible dynamic laws that apply on the systems micro-scale. The first one appears to be the famous paper by Fermi, Pasta and Ulam [11]. Roughly speaking the results indicate that equipartition of temperature or energy is not easily obtained in these simulations whereas the second law is believed to be universal. In judging the simulation outcomes one has to realize that the second law of thermodynamics applies only on a large enough space and time scale. Therefore the second law is an emergent property. The "human condition" is to experience thermodynamic phenomena on these large enough space and time scales. Probably because it is an emergent property the second law has puzzled and still is puzzling scientists. If the second law of thermodynamics seems not to be satisfied in simulation and experiment an important question to raise is whether the space and time scales considered are large enough. For example ectropy (entropy) fluctuations [7] are not violations of the second law. They occur only on a small enough space and time scale and have been confirmed experimentally [8]. The occurrence at small time and space scales of what are called "second law violations" in [7], as well as the ability to quantify them, are consistent with the second law being an emergent property, the emergence being quantifiable, as shown in this paper. As opposed to [7] and most publications concerning the second law of thermodynamics our results are obtained without invoking probabilistic descriptions and measures. Our measure is ectropy and all our results are obtained from dynamic responses dictated entirely by the simple reversible deterministic mass-spring dynamics. The results themselves are partly stated in terms of things being either highly likely or unlikely. On a large enough time and space scale these things are either always respectively never observed in physics.

Although statistical mechanics has turned out valuable to help develop and understand thermodynamics we believe it also introduces difficult unnecessary problems like the ergodic hypothesis and satisfying equilibrium conditions. This is because statistical mechanics is not a physically realistic manner to describe systems at the micro-scale. One may argue that masses and springs are neither but we believe their simple, conservative, reversible dynamic behavior much better represents what is truly happening on the micro-scale of physical systems. As demonstrated in this paper this behavior alone explains the second law and its emergent nature. The explanation is largely due to the macroscopic scale which causes averaging to become a dominant phenomenon. To see this consider Fig. 2 extended with many more mass-spring pairs and associated normal modes. Also consider (5.6). As the number of mass-spring pairs and normal modes goes to infinity, in Fig. 2 and through (5.6) averaging causes energy equipartition among identical macroscopic system parts that contain an (almost) equal number of mass-spring pairs that also tends to infinity. If 
the second law of thermodynamics is directly responsible for the arrow of time we must also conclude from this paper that the arrow of time is also an emergent property obtained from the simple reversible system dynamics on the micro-scale.

To find and demonstrate emergent properties we need to increase sufficiently the scale of the system. Large scale systems tend to develop largely different time-scales on which phenomena take place. This causes numerical integration of these systems to become very inefficient and also inaccurate. The use of conservative linear (massspring) systems that are diagonalizable enabled us to partly circumvent these problems. They allow for efficient accurate partly analytic computation of time-averaged energies and the detection of total equipartition at arbitrary scales. This allowed us to raise the scale sufficiently while performing our computations on an ordinary PC.

Clearly our results could be criticized on the grounds of restricting ourselves to one dimensional mass-spring systems with identical masses and spring constants. The question is to what extend they are representative for physical systems to which the second law applies. On the other hand the mass-spring systems have normal modes that do not exchange energy. From this point of view our mass-spring systems constitute a worst case for the second law of thermodynamics to apply. In the future we plan to investigate large scale dynamical mass-spring systems with unequal masses and spring constants and macroscopic system parts having different compositions.

The major contribution of this paper is to show that the second law of thermodynamics emerges in a straightforward manner out of the simple, reversible deterministic dynamics of masses and springs that represent the micro-scale of a large scale linear dynamical system. To obtain this result the recognition that: (1) the second law is an emergent property, (2) ectropy is a measure of how well the second law is satisfied, and (3) time-averaging is a way to ensure a sufficiently large time-scale was crucial. Then the result was obtained thanks to (1) the large scale linear conservative mass-spring system provides analytical solutions for time-averaged energies (2) to show the emergence a moderate system size suffices. The result also relies on (3) a computer experiment that computes many dynamic responses of a large scale system starting from initial conditions with approximately identical ectropy values but otherwise random. From it we concluded that although at each ectropy level we can construct infinitely many states violating the second law, together these make up a negligible part of all possible states realizing this ectropy level. This explains why Loschmidt's paradox is not a paradox when taking the scale large enough. This result also confirms the well known argument that although any isolated large scale conservative system behaves cyclic in principle, the cycle time is so large that no cycle is ever observed in physical reality [10].

The extension of our results to other physical systems having unequal parts at the micro-scale but linear dynamics will most likely require us to raise the scale and with it the number of computations significantly. We speculate that to obtain sufficient averaging the scale must be increased with an order of magnitude for each difference at the micro-scale. As long as the system is linear and diagonalizable we can still use the associated analytic responses and time-averages that prevent us from having to perform numerical integration that is time-consuming and inaccurate, especially for large scale systems. If the system becomes nonlinear in general it seems that this can no longer be prevented. 
Concerning Maxwell's Demon, the best advocate of the puzzle, but also a construct of human imagination, we would finally like to comment. This paper revealed that the second law of thermodynamics is an emergent property that relies entirely on the simple "ignorant" reversible dynamics of system parts at the micro-scale. Maxwell's Demon however is "aware" of the emergent property and tries to exploit this awareness to beat the second law of thermodynamics. So Maxwell's Demon would be a dynamic system on the micro-scale designed using "macroscopic information". In this respect the dynamics of Maxwell's Demon would be radically different from all the other simple "ignorant" ones that determine the emergence of the second law. If it would be possible to physically realize Maxwell's Demon this would radically change what happens on the micro-scale. But even then analysis of energy and information flows associated to an imagined physical realization of Maxwell's Demon still seem to rule out the possibility to beat the second law [10, 12]. Also recall that beating the second law implies beating it on a large enough (macroscopic) time and space scale to which it applies. So we conclude that Maxwell's Demon remains a construct of human imagination.

Open Access This article is distributed under the terms of the Creative Commons Attribution Noncommercial License which permits any noncommercial use, distribution, and reproduction in any medium, provided the original author(s) and source are credited.

\section{References}

1. Willems, J.C.: Book review. IEEE Trans. Autom. Control 51(7), 1217 (2006)

2. Haddad, W.M., Chellaboina, V.S., Nersesov, S.: Thermodynamics: A Dynamical Systems Approach. Princeton Series in Applied Mathematics. Princeton University Press, Princeton (2005)

3. Bernstein, D.S., Bhat, S.P.: Energy equipartition and the emergence of damping. In: Proceedings of the 41st IEEE Conference on Decision and Control, Las Vegas, Nevada, December, p. 2913 (2002)

4. Bhat, S.P., Bernstein, D.S.: Average-preserving symmetries and equipartition in linear Hamiltonian systems. In: 43rd Conference on Decision and Control, Atlantis, Paradise Island, Bahamas, 14-17 December, p. 2155 (2004)

5. Rapisarda, P., Willems, J.C.: Conserved- and zero-mean quadratic quantities in oscillatory systems. Math. Control Signals Syst. 17, 173 (2005)

6. Patrascioiu, A.: The Ergodic Hypothesis: A Complicated Problem in Mathematics and Physics. Los Alamos Science Special Issue, vol. 263 (1987)

7. Evans, D.J., Searles, D.J.: The fluctuation theorem. Adv. Phys. 51(7), 1529 (2002)

8. Wrang, G.M., Sevick, E.M., Mittag, E., Searles, D.J., Evans, D.J.: Experimental demonstration of violations of the second law of thermodynamics for small systems and short time scales. Phys. Rev. Lett. 89(5), 50601 (2002)

9. Lamb, J.S.W., Roberts, J.A.G.: Time-reversal symmetry in dynamical systems: a survey. Physica D 112, 1 (1998)

10. Von Bayer, H.C.: Warmth Disperses and Time Passes. The Modern Library, New York

11. Fermi, E., Pasta, J., Ulam, S.: Studies of nonlinear problems. In: Ulam, S. (ed.) Sets, Numbers and Universes. MIT Press, Cambridge (1974). (See also Los Alamos Scientific Laboratory report LA-1940 (1955))

12. Duncan, T.L., Semura, J.S.: Information loss as a foundational principle for the second law of thermodynamics. Found. Phys. 37, 1767 (2007) 